Article

\title{
Development and Testing of Novel Applications for Adsorption Heat Pumps and Chillers ${ }^{\dagger}$
}

\author{
Xavier Jobard *(D), Pierryves Padey, Martin Guillaume, Alexis Duret and Daniel Pahud \\ School of Management and Engineering Vaud, University of Applied Sciences and Arts Western Switzerland, \\ Yverdon-les-Bains 1400, Switzerland; pierryves.padey@heig-vd.ch (P.P.); martin.guillaume@heig-vd.ch (M.G.); \\ alexis.duret@heig-vd.ch (A.D.); daniel.pahud@heig-vd.ch (D.P.) \\ * Correspondance: xavier.jobard@heig-vd.ch; Tel.: +41-24-557-28-17 \\ + This paper is an extended version of our paper published in the Proceeding of ECOS 2019, the 32nd \\ International Conference on Efficiency, Cost, Optimization, Simulation and Environmental Impact of Energy \\ Systems, June 23-28 2019, Wroclaw, Poland.
}

Received: 29 December 2019; Accepted: 26 January 2020; Published: 1 February 2020

check for updates

\begin{abstract}
This work aims at the development and the experimental characterization of new applications for adsorption heat pumps and chillers driven by industrial waste heat or renewable sources that can provide heating and/or cooling. Adsorption technologies offer the advantage of providing heating and cooling from low temperature sources below $100{ }^{\circ} \mathrm{C}$ without using refrigerant with high Global Warming Potential and with very low electricity consumption. Therefore, the technology enables the use of large untapped heat sources, increasing the energy efficiency of the heating and cooling sector with very limited impact on the environment. Several applications were investigated numerically for Switzerland using a simplified model of an adsorption heat pump. Four scenarios were identified as interesting: (1) the valorization of low-grade industrial waste heat in district heating networks, (2) energy efficiency improvement of district heating substations, (3) an autonomous adsorption heat pump with a wood pellets burner and (4) cooling applications. These scenarios were experimentally validated with a laboratory test of a commercial silica gel/water machine. Results show that there is a gap of up to $40 \%$ between the prediction of the simplified model and the experimental results. Therefore, there is huge potential to improve the performances of this commercial unit for these applications.
\end{abstract}

Keywords: adsorption heat pump; district heating network; adsorption chillers; waste heat recovery; energy efficiency.

\section{Introduction}

According to [1], the energy required for cooling in Switzerland already makes up $11 \%$ of Swiss electricity consumption, representing around $6000 \mathrm{GWh}$ annually. An important share of this electricity consumption (1200 GWh) is used for air conditioning. In addition, due to the increase in extreme temperatures in summer, the share of electricity used for air conditioning will increase significantly in the near future. Furthermore, the share for space heating is even larger and represents $33 \%$ of the Swiss final energy consumption. A large portion of this energy $(>70 \%)$ is produced with fossil fuels (natural gas and oil) which are responsible for greenhouse gas emissions. However, industrial waste heat has a very important and untapped potential. In Switzerland, it represents around 170 TWh every year [2]. For comparison, the 2011 Swiss space heating and cooling demand amounted to 65 and 28 TWh respectively. Valorizing industrial waste heat for space heating or cooling demand would thus be an interesting option to fulfill the Swiss energy turnaround strategy [3] and reduce the stress on the electric production system and grid. 
One possible solution for such valorization goals is the use of an adsorption heat pump (adHP). An adHP is a thermally driven heat pump which uses the adsorption process to produce heating or cooling [4]. AdHP using water for the refrigerant can supply cooling from $0{ }^{\circ} \mathrm{C}$ and more and heating from $20^{\circ} \mathrm{C}$ to $60^{\circ} \mathrm{C}$ or more depending on the driving temperature and adsorbent. AdHP technologies have a relatively long history: starting with Faraday in 1823 [5] they were relatively widely used for cooling and air conditioning in the first half of the 20th century. Due to the availability of cheap electric motors, vapor compression technology was then preferred. Nevertheless, since the 1990s, a renewed interest in adHP has been observed, due to the rising cost of energy and to environmental concerns (global warming and ozone layer depletion). Thereby, recently at least two important European companies (Viessmann [6] and Vaillant [7]) released gas fired adsorption heat pumps for heating and domestic hot water production on the market. Moreover, adsorption refrigeration technology is being and has been widely studied for solar cooling and air conditioning [8]. Small-scale commercial units (cooling power $<50 \mathrm{~kW}$ ) for these applications are available in Europe from at least three manufacturers (Fahrenheit, Invensor and Solabcool).

AdHP technology could contribute to the Swiss energy panorama because it offers several advantages over conventional mechanical vapor compression heat pumps [4]. It has very low electricity consumption (high electrical COP) and can use natural refrigerants, such as water, which have no ozone depletion effect, minimal global warming potential (GWP), and are cheap and available. Energy can be supplied with various heat sources (e.g., waste-heat, solar thermal energy). Moreover, since adHP has no moving parts, it has long lifetime and requires only little maintenance. However, this technology suffers also from several drawbacks, e.g., because of its discontinuous cycle. Therefore, several adsorber beds have to be used in parallel for continuous heating/cooling production. In addition, it has a relatively low thermal COP and a low power density compared to mechanical heat pumps. However, adHP does not benefit from the same technology maturity as compression HP, hence the cost remains high and the efficiency low.

A key feature of adHP technology is that the driving energy may be low-grade heat from various sources such as waste heat from industrial processes or waste incineration, district heating networks (DHN), cogeneration, solar thermal or gas burners. Thus, adHP could contribute to the Swiss energy efficiency objectives and reduce its greenhouse gas emission by offering the possibility to valorize waste heat efficiently to satisfy a part of the heating and cooling demand.

Recently, researchers focused on the development of new adsorptive transformation devices. A new cycle for the heat transformation of ambient air at negative temperatures was presented by Aristov [9] and tested by Tokarev [10]. Freni et al. [11] report advanced sorbent materials such as new zeolite-like materials (AIPO and SAPO) and composite adsorbents like $\mathrm{LiBr}$-silica and $\mathrm{CaCl}_{2}$-silica as very promising for conventional adsorptive heat pumps and chillers. In parallel of the enhancement of the working pairs' performance, studies are carried out on the intensification of the heat and mass transfer [12-14]. These recent advances could open new fields of applications for adHP, because of enhanced performances for specific temperature levels.

In this context, this work aims at the definition of the most suitable application scenarios for adHP in Switzerland. Firstly, the methodological framework that has been developed in order to identify the scenarios is presented. Secondly, the constraints and boundary conditions are exposed, as well as a simple and new numerical model to estimate the performances of adHP with limited input data. Finally, the scenarios are validated with laboratory experiments and the results are discussed.

\section{Method to Identify Application Scenarios for adHP}

\subsection{Methodological Framework}

To reach the objectives of this study, which are to valorize low temperature heat with adHP to meet heating and cooling needs, the method exposed in Figure 1 was established. First, the temperature level, technical and thermodynamics constraints were defined to set the context of the application scenarios. 


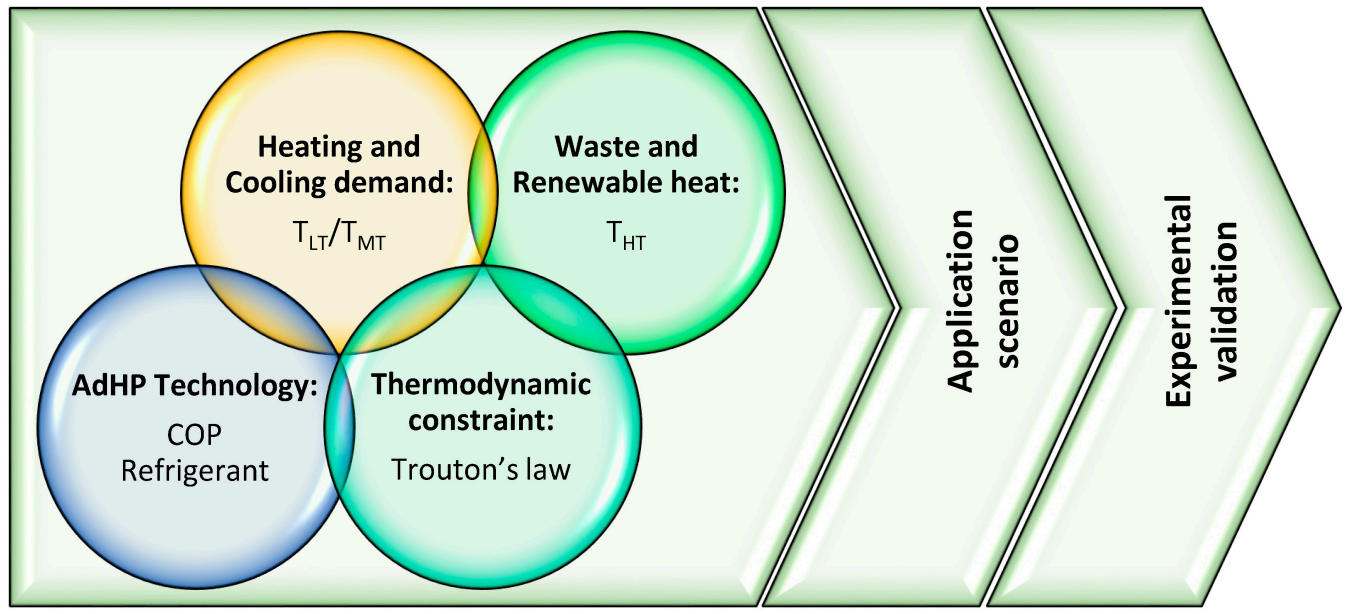

Figure 1. Diagram of the methodological framework for the elaboration and validation of the application scenarios of the adsorption heat pump (adHP).

Firstly, adHP require three heat sources at different temperature levels, a low temperature source $\mathrm{T}_{\mathrm{LT}}$, a medium temperature source at $\mathrm{T}_{\mathrm{MT}}$ and a high temperature source $\mathrm{T}_{\mathrm{HT}}$ to operate, as shown in Figure 2. The temperature difference between the $\mathrm{T}_{\mathrm{HT}}$ and $\mathrm{T}_{\mathrm{MT}}$ provides the necessary work to drive a refrigeration cycle between the temperature $\mathrm{T}_{\mathrm{MT}}$ and $\mathrm{T}_{\mathrm{LT}}$. Therefore, heat is consumed at the temperature $\mathrm{T}_{\mathrm{HT}}$, heating energy is provided at $\mathrm{T}_{\mathrm{MT}}$ and cooling energy at $\mathrm{T}_{\mathrm{LT}}$. These three temperatures are defined by the law of thermodynamics and specifics are detailed in Section 2.2.

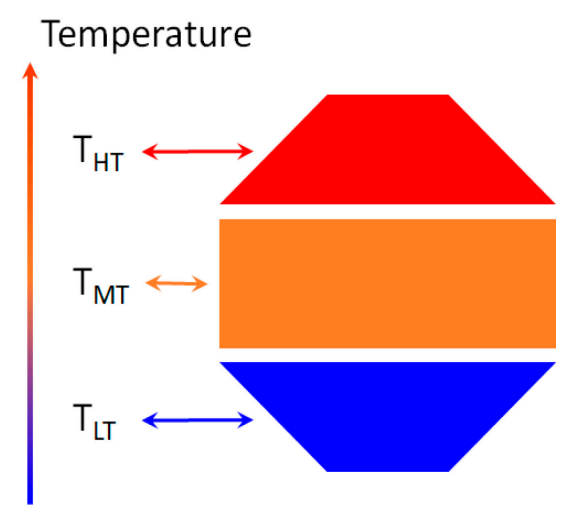

Figure 2. Schematic of a trithermal adsorption device

The temperature levels of the heat sources and heat sinks were estimated based on the authors' knowledge and on available literature:

- The space heating temperature levels are estimated based on a study reporting on the heating distribution systems of the Swiss building stock [15]

- The cooling demand temperatures are usually found for air conditioning as well as for the conditioning of data centers.

- Heat sources were limited to temperatures lower than $100{ }^{\circ} \mathrm{C}$ to maximize waste heat recovery and renewable heat utilization.

Secondly, to guarantee the interest of the scenarios, technical constraints were set in terms of targeted energy efficiency and environmental performances. These are translated in the use of water as the refrigerant excluding $\mathrm{T}_{\mathrm{LT}}$ under $0^{\circ} \mathrm{C}$ and a threshold on the thermal coefficient of performance (COP). The temperature levels of the thermostats influence the COP of the adHP. Therefore, a numerical 
model is required and was developed (see Section 2.3) to estimate the COP in function of the considered temperatures.

Based on this model and the reported constraints, the most suitable scenarios for adHP applications are proposed in Section 3, and their interest and characteristics are discussed. Finally, each scenario is experimentally validated. Hence, lab-tests were run in order to quantify the real performances of the adHP with the boundary conditions defined by the scenarios. The experimental results are discussed in two folds: 1 . the numerical model is analyzed in perspective to the lab-tests and 2. the experimental results are discussed and perspectives for the development of the technology are given.

This integrated approach, from the adHP performance model to the scenario definition and finally to the practical adHP performance, enables a complete and integrated vision for the large-scale deployment of the technology in Switzerland.

\subsection{Thermodynamics Constraints}

As presented previously, an adHP cycle operates between three different temperature levels: $T_{H T}$, $T_{M T}$ and $T_{L T}$ as shown in Figure 2. As stated in [16], the maximum intermediate temperature $T_{M T}$ is a function of $T_{L T}$ and $T_{H T}$. The relation has been called Trouton's limit in [17] and is expressed as:

$$
T_{M T}^{2} \leq T_{H T} \cdot T_{L T}
$$

The demonstration of the so-called Trouton's limit, expressed in the Equation (1) can be found in [18]. For this paper the maximum $T_{M T}$ temperature that can be achieved will be called the Trouton's temperature, expressed as $T_{\text {Trouton }}$.

This work aims at considering industrial waste heat (or renewable energy) as a driving source and eventually as a cold source, as well as from wasted heat or heat from the environment. Thus, based on the refrigerant choice (water) and the fact that only low-grade industrial waste heat should be considered, the possible scenarios should be limited by $T_{H T}<373 \mathrm{~K}$ and $T_{L T}>273 \mathrm{~K}$.

Thereby, because of Trouton's limit, the possible application will have to consider a relatively low $T_{M T}$ temperature. Based on this observation, it has been identified that-among others-space heating for buildings could be an interesting solution for the valorization of the adHP energy.

The possible adHP application scenarios were developed taking all those constraints into account. A simple adHP numerical model had to be developed in order to ensure that the proposed solutions would have the proper performances in terms of COP and operating temperatures.

\subsection{Numerical Modelling of AdHP Technical Performances}

The study aimed at developing application scenarios for which the adHP machine should obtain a coefficient of performance (COP) above 1.45 for heating $\left(\mathrm{COP}_{h}\right)$, and 0.45 for cooling $\left(\mathrm{COP}_{c}\right)$. These thresholds were set during the project initial phase, in order to find new scenarios for the adHP with performance in the range of the current existing machines. A numerical model is thus needed to estimate the COP of the adHP for different temperatures. The current available numerical models to assess the performances of adHP are classified in three categories according to the [19]: (i) thermodynamic model, (ii) lumped-parameter model, (iii) distributed-parameter model. On the one hand, the last two categories require a large amount of data related to the design (i.e., exchanger heat transfer coefficient), operation (i.e., cycle time) and physical properties (i.e., heat of adsorption) of the device, but are able to model accurately the dynamics of the adHP according to the complexity of the model. On the other hand, thermodynamic models essentially rely on the application of the first and second law of thermodynamics and require an equilibrium isotherm to calculate the vapor uptake [19]. In the end, they do not take into account the technical irreversibilities, and therefore tend to overestimate the performances of adHP. For this reason, a simple regression model of the technical efficiency based on an extensive literature review was developed for this work and is presented here after. 
First, the ideal Carnot COP of the adHP is function of the operative temperature triplet and is given by:

$$
\begin{gathered}
\text { Heating : } \operatorname{COP}_{h}^{C} \equiv Q_{M T} / Q_{H T}=\left(\frac{1}{T_{L T}}-\frac{1}{T_{H T}}\right) /\left(\frac{1}{T_{L T}}-\frac{1}{T_{M T}}\right) \\
\text { Cooling : } C O P_{c}^{C} \equiv Q_{L T} / Q_{H T}=C O P_{h}^{C}-1
\end{gathered}
$$

Equations (2) and (3) describe the ideal performance of the adHP devices, but in practice, due to irreversilibities and non-ideal characteristics, the adHP devices have lower COPs. The ratios between real COP and Carnot COP define the heating and cooling technical efficiency, also referred to as thermodynamic efficiency and expressed as:

$$
\begin{aligned}
& \text { Heating: } \eta_{\text {tech }_{h}}=\mathrm{COP}_{h} / C O P_{h}^{C} \\
& \text { Cooling: } \eta_{\text {tech }_{c}}=C O P_{c} / C O P_{c}^{C}
\end{aligned}
$$

In order to estimate the so-called technical efficiency of the current adHP machine, the literature review has been carried out considering scientific publications $[4,20,21]$ as well as the technical performance of commercialized adHP devices. Based on the retrieved datasets, a regression model (6) was developed on the base of a $\Delta T_{\text {ratio }}$ (7) inspired from [22] and the difference between the Trouton temperature limit and the $T_{M T}$ temperature. The temperature triplets, as well as the experimental COP, were collected and the parameters in (6) were identified by minimizing the error between the model and the datasets comprising 132 temperature triplets. The resulting coefficient of correlation of this model compared with the data sample is very satisfying and reaches 0.86 with a Root Mean Square Error (RMSE) of 0.05 and a Mean Absolute Percent Error (MAPE) of $8.8 \%$.

$$
\begin{gathered}
\eta_{\text {tech }}=0.74+0.31 \times \ln \left(\Delta T_{\text {ratio }}\right)+3.6 \times 10^{-3} \times\left(T_{\text {Trouton }}-T_{M T}\right) ; R M S E=0.05 \\
\Delta T_{\text {ratio }}=\frac{T_{M T}-T_{L T}}{T_{H T}-T_{M T}}
\end{gathered}
$$

where: $T_{\text {Trouton }}^{2}=T_{H T} \cdot T_{L T} ;\left[K^{2}\right]$ is maximum temperature of the intermediate source according to Trouton's limit.

Different other fitting models have been tested such as multiple regression, Principal Component Regression (PCR), Partial Least Square regression (PLS) and Multivariate Adaptative Regression Spline (MARS), the RMSE and MAPE are given in the Table 1.

Table 1. Comparison of the RMSE and MAPE for different fitting models.

\begin{tabular}{ccc}
\hline Fitting Approach & RMSE & MAPE \\
\hline Developed model (Eq. 6) & 0.050 & $8.8 \%$ \\
Multiple Regression & 0.061 & $10.8 \%$ \\
PCR & 0.092 & $17.5 \%$ \\
PLS & 0.080 & $14.6 \%$ \\
MARS & 0.044 & $7.1 \%$ \\
\hline
\end{tabular}

Only the MARS model provides better RMSE and MAPE results. Nevertheless, the MARS model has no physical significance. Considering the low level of improvement for the fitting adequacy, the model developed in the equation (6) has been kept. Within this model, it appears that when the difference between the $T_{M T}$ temperature and the $T_{\text {Trouton }}$ is large, the technical efficiency is increased. Conversely, the higher the $\Delta T_{\text {ratio }}$ is, the higher the technical efficiency is.

Finally, it has to be noticed that the developed model has been set considering water for the adsorbate whereas various adsorbents were considered (silica and zeolite). While the adsorbent materials probably play a role in the COP depending on the temperature triplet, the model estimating 
their technical efficiency has been kept only with the temperatures as variable since the model aims at identifying the possible new operating scenarios for adHP.

\subsection{AdHP Experimental Characterization}

Once the scenarios are defined an experimental validation was realized. This validation aimed at ensuring the relevance of the scenarios. To do so, a commercial adHP has been characterized on a test bench specially designed to test thermally driven heat pumps. This commercial device was operated out of its normal temperature ranges because of the unusual temperature produced by the scenario definition.

\subsubsection{Test Bench Description}

The test bench allows for performing dynamic tests of air conditioning systems, heating systems, solar cooling systems and tri-thermal pumps. It consists of three separate loops, filled with 40/60 mono-propylene-glycol/water mixture, that can be conditioned to different temperature levels and flow rates (see Figure 3).

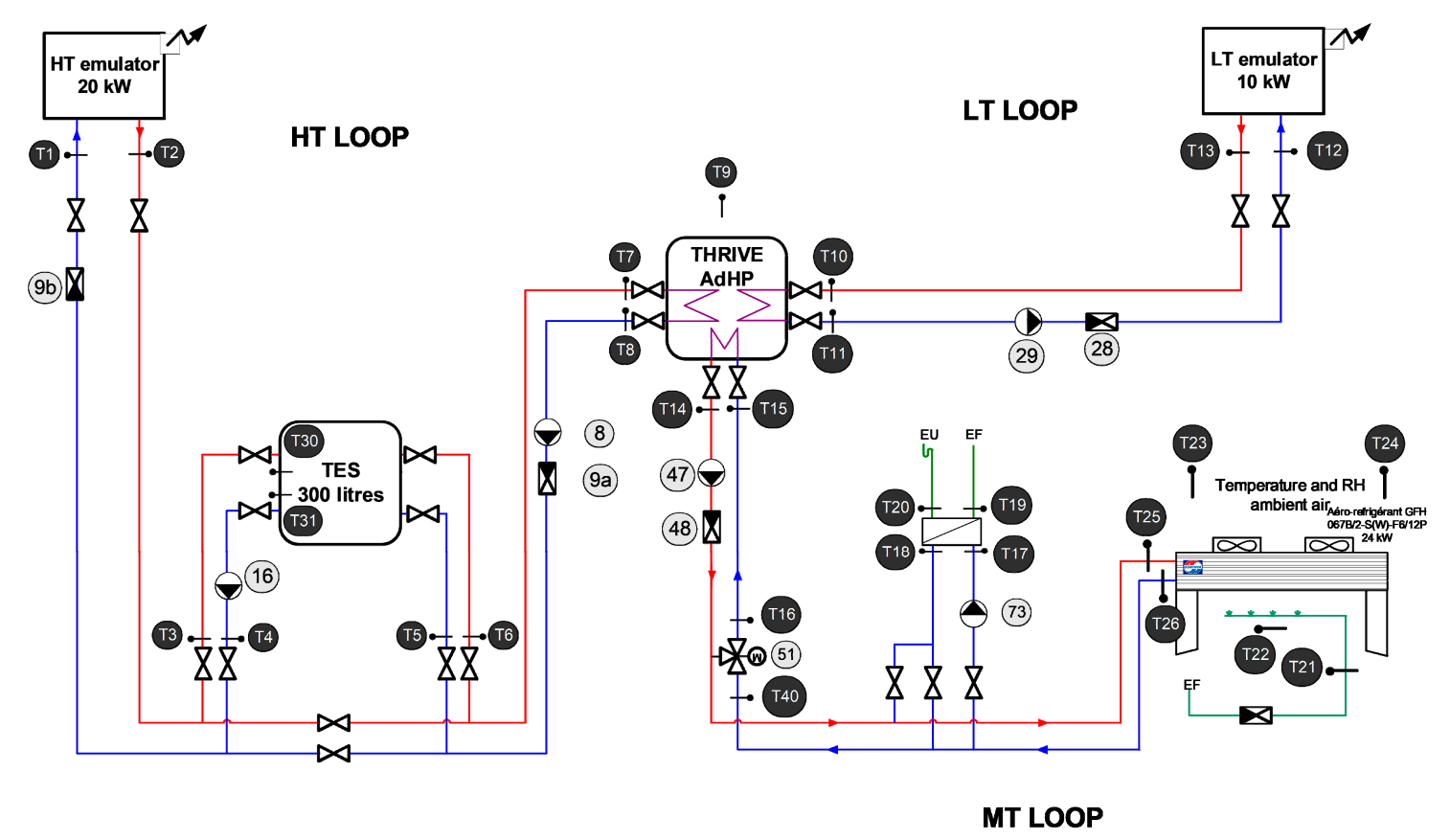

Figure 3. Hydraulic Scheme of the adHP test bench.

On the high temperature (HT) loop, an immersed electric heater with a power of $20 \mathrm{~kW}$ emulates the heating source. A thermal energy storage tank with a capacity of 3001 is installed on the HT loop, to obtain better stability of the HT adHP inlet temperature. Hydraulic connections also allow bypassing this tank, and the heat transfer fluid (HTF) flows then directly from the emulator to the adHP. A 10kW electric heater installed on the low temperature (LT) loop emulates the chilled water consumption or cold source. The adHP inlet temperature is set according to the cooling application or chosen heat sink. The adHP medium temperature (MT) inlet is controlled with a recooler composed of a dry cooler supplemented with sprinklers and a mixing control valve. The recooler has a nominal power of $24 \mathrm{~kW}$. An additional heat exchanger fed with tap water, installed on the return line between the recooler and the adHP, also increases the cooling capacity of the MT loop and provides better control of the return temperature to the adHP, regardless of the weather conditions. 


\subsubsection{Test Object}

In this work, an eCoo10 machine from the FAHRENHEIT company was installed and tested on the described test bench. This silica gel-water adsorption chiller has a nominal cooling power of up to $16.7 \mathrm{~kW}$. Further characteristics are to be found in Table 2.

Table 2. Characteristics of the Fahrenheit eCoo 10 machine tested on the test bench.

\begin{tabular}{cccc}
\hline \multirow{3}{*}{ General Characteristics } & Cooling Capacity & Up to 16.7 & $\mathrm{~kW}$ \\
& COP & Max. 0.65 & - \\
& Electrical power consumption & 260 & $\mathrm{~W}$ \\
\hline \multirow{2}{*}{ Hot water circuit } & Application range & $50-95$ & ${ }^{\circ} \mathrm{C}$ \\
& Nominal volume flow rate & 2500 & $\mathrm{l} / \mathrm{h}$ \\
\hline \multirow{2}{*}{ Re-cooling circuit } & Application range & $22-40$ & ${ }^{\circ} \mathrm{C}$ \\
& Nominal volume flow rate & 5100 & $1 / \mathrm{h}$ \\
\hline \multirow{2}{*}{ Chilled water circuit } & Application range & $8-21$ & ${ }^{\circ} \mathrm{C}$ \\
& Nominal volume flow rate & 2900 & $1 / \mathrm{h}$ \\
\hline
\end{tabular}

The eCoo 10 consists of two chambers each containing an adsorption/desorption chamber and an evaporator/condenser, that allows producing continuous cooling. The eCoo 10 operates according to the four following phases as described by the manufacturer:

- Phase 1: Desorption/Adsorption Phase

During this phase, the HT loop is connected to the chamber where desorption is occurring. The temperature rise of the chamber desorbs the water accumulated in the silica gel. The MT loop, which is connected to the condenser, recovers the heat of the water condensation. At the same time, in the other module, the water is evaporated in the evaporator with LT loop heat, producing the cooling effect. Water vapor is then adsorbed on the sorption material releasing heat of adsorption. The MT loop connected to the chamber where adsorption is operating, recovers this heat of adsorption.

- $\quad$ Phase 2: Heat Recovery Phase

Once the desorption/adsorption phase is finished, the heat recovery phase starts. Its main purpose is to recover a part of the parasite sensible heat stored in the sorption module itself. The MT loop is connected to inlet of the chamber where the desorption was operating (hot chamber) but the outlet is still connected to the HT loop. The heat stored in this reactor is consequently moved to the HT loop. The inlet of the $2^{\text {nd }}$ chamber, where the adsorption was previously operating (cool chamber), is connected to the HT loop. The outlet of this chamber-in which the desorption process is starting-is still connected to the MT loop for a defined amount of time.

- $\quad$ Phase 3: Heat Recovery Phase, and 4: Desorption/Adsorption Phase:

These phases are identical to phase 1 and 2 but the adsorption and desorption chambers as well as the evaporator and the condenser are interchanged.

While in principle the machine performances are characterized by the product, the current project developed non-traditional scenarios for which the machine performances were unknown and had to be measured to verify if the application was relevant or needed material development to be implemented.

\subsubsection{Measurements}

Specifications on the test procedures for sorption chiller were developed during the IEA SHC Task 48 [23] and are used here to guarantee reliable and reproducible results. Therefore, the adHP was tested in stationary conditions: the inlet temperatures and flowrates were kept constant with 
a maximum standard deviation of $0.2 \mathrm{~K}$ for the temperatures and $2 \%$ for the flowrates. From the measurements of the instantaneous power $\dot{Q}[\mathrm{~W}]$ of each loop $x$ (with $=[\mathrm{LT}, \mathrm{MT}, \mathrm{HT}]$ ) calculated,

$$
\dot{Q}_{x}=\dot{v}_{x} \cdot \rho . c p \cdot\left(\theta_{x, i}-\theta_{x, o}\right)
$$

the energies transferred during these two consecutive cycles calculated by integrating the instantaneous power are computed as follows:

$$
Q_{x}=\int \dot{Q}_{x}(t) \cdot d t
$$

Then, the mean power $\overline{\dot{Q}}_{x}$ (or rated power) representative of the tested operating point obtained by dividing the energy $Q_{x}$ by the total duration of the two considered cycles $\tau_{2 c}$ :

$$
\overline{\dot{Q}}_{x}=Q_{x} / \tau_{2 c}
$$

The efficiency of adHP machines is quantified with the dimensionless number, the thermal COP used for heating $\left(C O P_{h}\right)$ and for cooling $\left(C O P_{c}\right)$. The thermal heating $C O P$ represents the ratio between the produced heating energy and the supplied driving thermal energy. The thermal COP for cooling represents the ratio between the produced cooling energy and the supplied driving thermal energy.

$$
C O P_{h}=Q_{\mathrm{MT}} / \mathrm{Q}_{\mathrm{HT}} ; C O P_{c}=\mathrm{Q}_{\mathrm{LT}} / \mathrm{Q}_{\mathrm{HT}}
$$

The relative uncertainties related to the measurements made on the test bench is calculated with a coverage factor of $\mathrm{k}=1$. This same factor is used to calculate the uncertainty on the instantaneous power/energy flows through the adHP. The absolute uncertainties for the temperature and the flow rates are presented in Table 3. The relative uncertainty on the HTF specific heat capacity and density product is considered to be $1 \%$.

Table 3. Summary of the absolute uncertainties of the calibrated temperature and flowmeters with a coverage factor of $\mathrm{k}=1$.

\begin{tabular}{cccccccccc}
\hline & $\delta \theta_{L T, i}$ & $\delta \theta_{L T, o}$ & $\delta \theta_{M T, i}$ & $\delta \theta_{M T, o}$ & $\delta \theta_{H T, i}$ & $\delta \theta_{H T, o}$ & $\delta \dot{v}_{L T}$ & $\delta \dot{v}_{M T}$ & $\delta \dot{v}_{H T}$ \\
\hline Unit & ${ }^{\circ} \mathrm{C}$ & ${ }^{\circ} \mathrm{C}$ & ${ }^{\circ} \mathrm{C}$ & ${ }^{\circ} \mathrm{C}$ & ${ }^{\circ} \mathrm{C}$ & ${ }^{\circ} \mathrm{C}$ & $1 / \mathrm{h}$ & $1 / \mathrm{h}$ & $1 / \mathrm{h}$ \\
\hline Uncertainty $(\mathrm{k}=1)$ & 0.020 & 0.016 & 0.020 & 0.036 & 0.033 & 0.018 & 1.95 & 13.67 & 7.26 \\
\hline
\end{tabular}

The uncertainties propagation is calculated according to the statistical method presented in [24] and presented in (12) for the function $f\left(x_{n}\right)$.

$$
\left(\delta f\left(x_{n}\right)\right)^{2}=\sum_{i=1}^{n}\left(\frac{\partial f}{\partial x_{i}}\right)^{2}\left(\delta x_{i}\right)^{2}
$$

Uncertainty on the energy produced, average power, and COPs are calculated with a coverage factor of $k=2$, giving a level of confidence of approximately $95 \%$. Applying the calculations to one operational condition of the adHP machine-where the resulting $C O P_{h}$ is 1.46 and the $C O P_{c}$ is 0.48 - the relative uncertainties are, respectively, $0.16 \%$ for the $C O P_{h}$ and $0.2 \%$ for the $\mathrm{COP}_{c}$.

\section{Application Scenarios}

\subsection{Scenario 1: Valorisation of Waste Heat for District Heating Grids}

Scenario 1 aims at recovering waste heat from two industrial sources within the return pipe of a district heating network, as shown in Figure 4 . For this scenario, a low temperature $\left(\theta_{L T}\right)$ industrial waste heat is coupled within the adHP to a high temperature industrial waste heat source $\left(\theta_{H T}\right)$. 
This scenario has been developed based on three observations: first, the industrial waste heat often is located near to the urban center. Second, creating a distribution network for valorizing the waste heat is costly and ensuring a synergy with existing distribution network is thus relevant. Finally, because of temperature demand for space heat and domestic hot water production (relatively high temperature, approx. $70 / 80^{\circ} \mathrm{C}$ for old building) the adHP will require a backup in order to fulfil the energy demand.

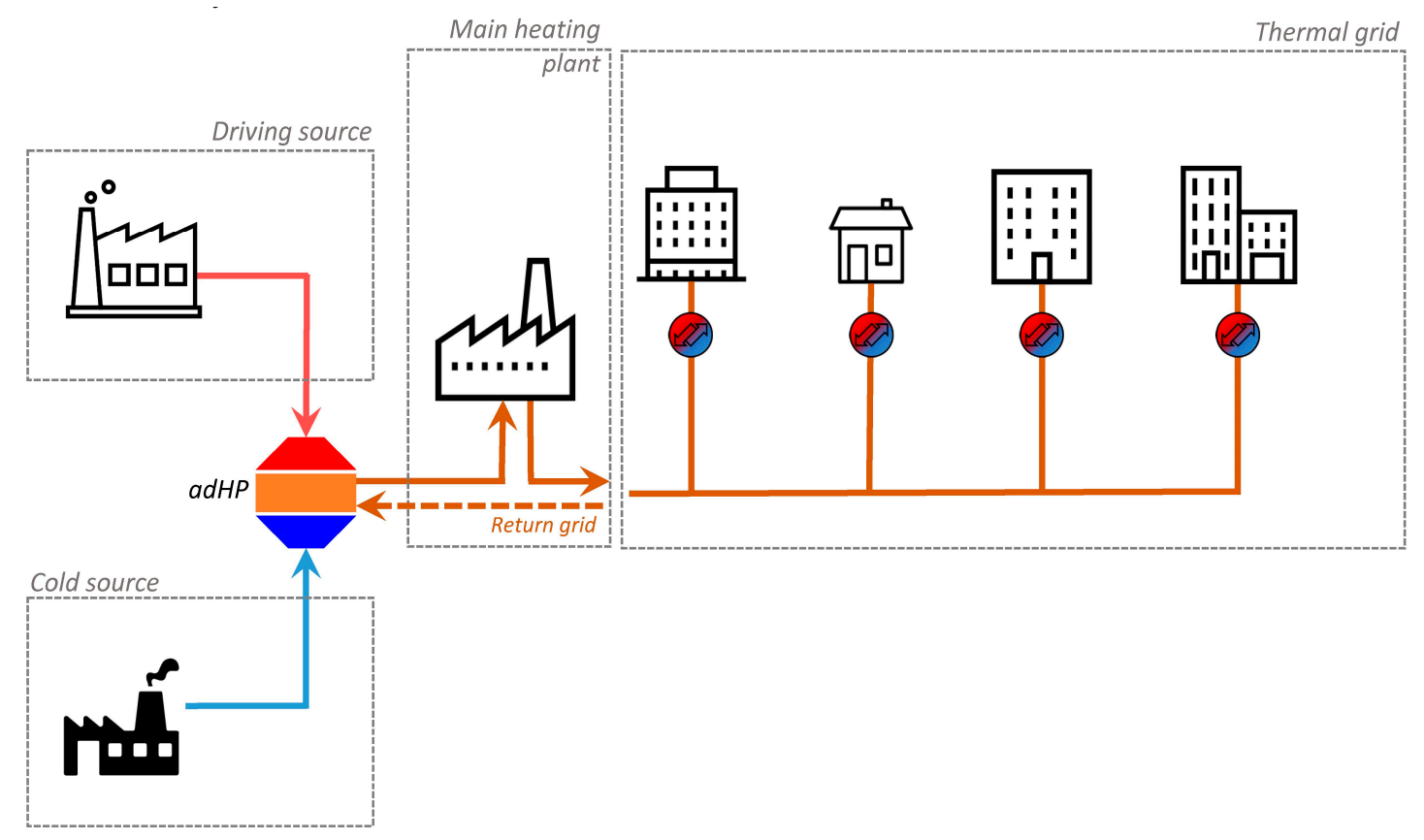

Figure 4. Schematic representation of Scenario 1.

Thereby, in this scenario, the adHP valorizes industrial waste heat and provides energy to a district heating network (DHN). Because of the adHP operative temperature, the energy is provided to the return pipe of the DHN and then, if necessary, the delta energy is provided by the main heating plant.

Thus, the return temperature of the district heating network has to be as low as possible in order to obtain the best performances for the adHP. Third to fourth generation DHNs [25] are relevant for the implementation of adHP in such a configuration. Depending on the temperature levels of the two heat sources valorized and on the thermal grid return/forward temperatures, the energy recovered and released by the adHP will be different.

In this scenario, the DHN temperature levels are thus critical, especially the return temperature. The lower the return temperature will be, the higher the adHP performance and share of energy will be. Based on this observation, several temperature triplets have been considered. The optimal triplet found as relevant is $=16^{\circ} \mathrm{C}, \theta_{M T}=40^{\circ} \mathrm{C}, \theta_{H T}=95^{\circ} \mathrm{C}$, since it represents an optimum in term of energy valorization on Swiss district heating networks, considering the practical real temperature levels. Based on the performance model, the estimated practical COP is estimated to be 1.48. The driving source is at the higher boundary of the machine constraints (see Section 2.1), the cold source is in the lower range while the intermediate temperature could provide a significant share of energy to a DHN operating at $60 / 30{ }^{\circ} \mathrm{C}$ (forward/return temperature), i.e, low temperature district heating network.

This scenario was found to be already interesting for some cases but its relevance should grow over time when the thermal grid temperature will be lowered. The proposed scenario considers a centralized application with a large adHP unit, valorizing large amounts of wasted heat within a district heating network. 


\subsection{Scenario 2: Improvement of the District Heating Network Substation Efficiency}

Scenario 2 proposes an innovative solution to decrease the return temperature of a DHN with a new substation design including an adHP. This concept has been defined initially by Zhang et al. [26], and the substation scheme is presented in the Figure 5:

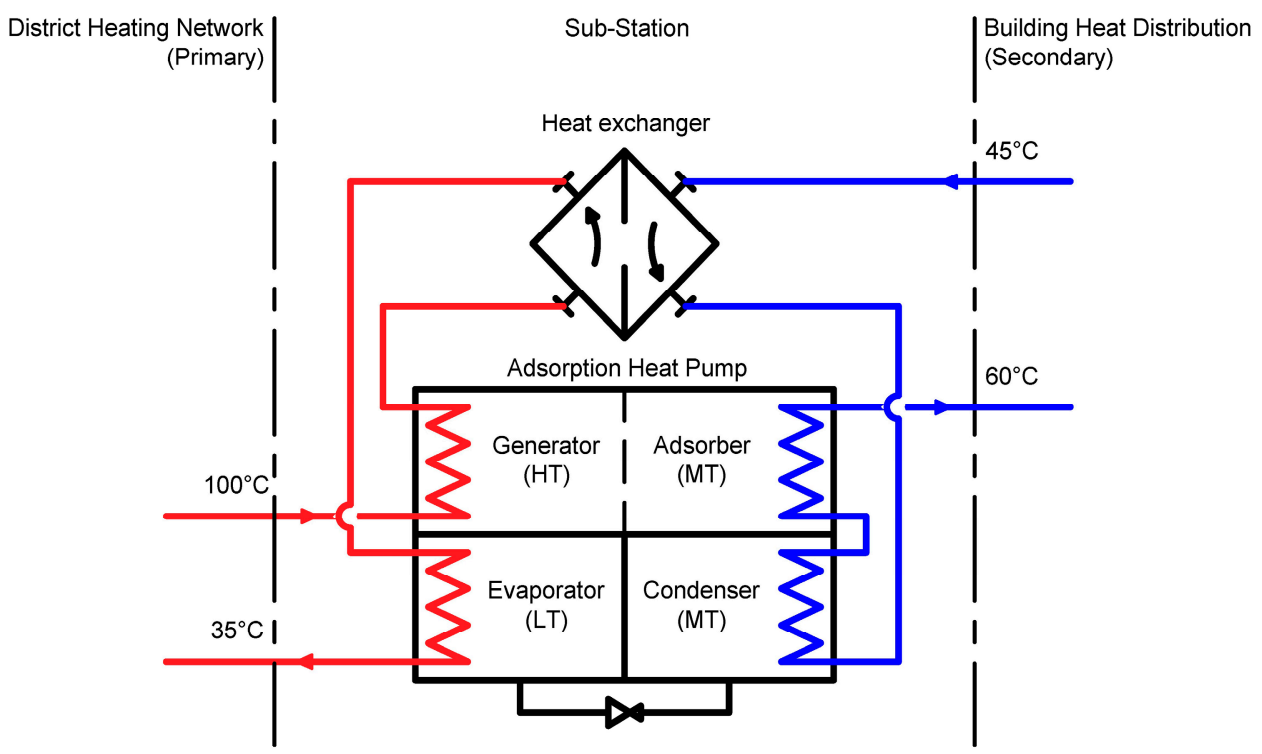

Figure 5. District heating substation including adHP, according to [26].

With this so-called "heat adaptor" substation architecture, the return temperature of the DHN could be decreased at a level that could be lower than the return temperature from the secondary loop (the heat distribution of the buildings), limiting the influence of the old energy-intensive buildings operating at high temperatures for the space heat distribution.

Thus, in this scenario, this new concept of a district heating substation would be installed in the buildings (decentralized application), giving priority to old buildings with high temperature distribution networks, as shown in Figure 6.

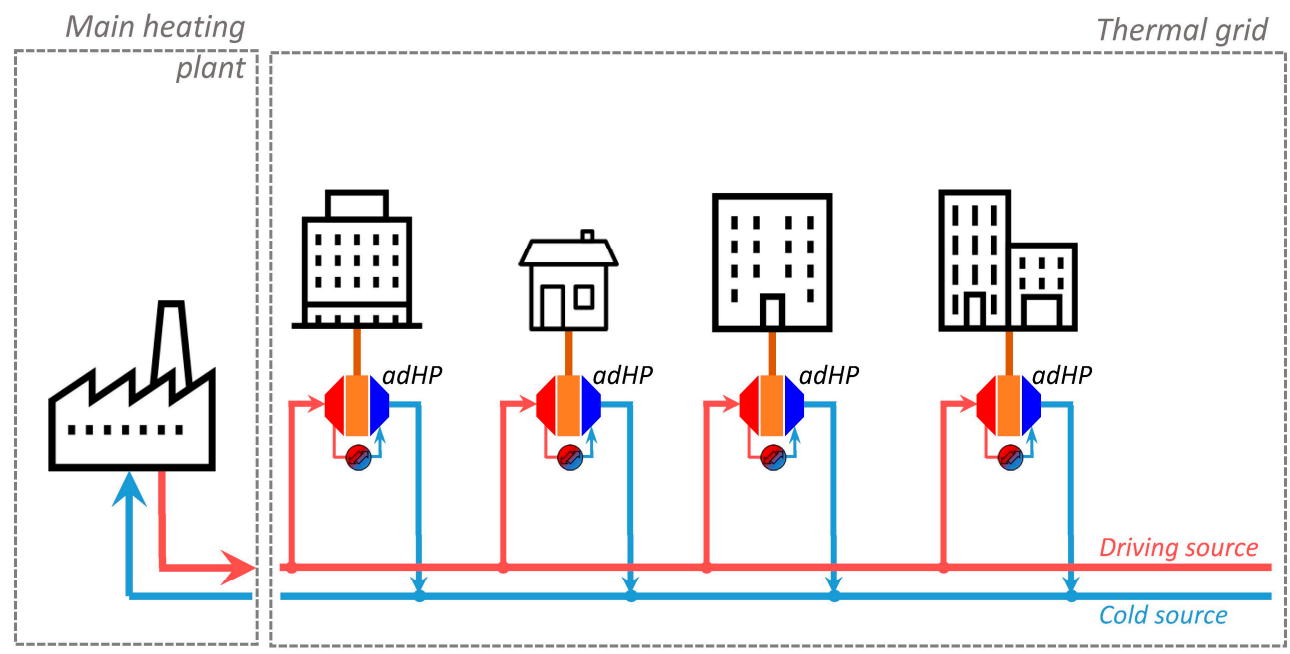

Figure 6. Schematic representation of Scenario 2.

By decreasing the thermal grid's return temperature, at constant flow rate, the grid capacity is increased. The heating plant efficiency could also benefit from this lower temperature; for example, 
by condensing the water contained in the flue gas, at the stack. At constant capacity, flow rates could also be reduced, leading to lower energy consumption of the circulating pumps and subsequently, higher efficiency of the heating plant.

In this scenario, the temperature triplet for the test phase is $\theta_{L T}=48^{\circ} \mathrm{C}, \theta_{M T}=58^{\circ} \mathrm{C}$, and $\theta_{H T}$ $=82^{\circ} \mathrm{C}$, leading to an estimated COP of 1.56 . This triplet has been considered by assuming a DHN operating at a forward temperature of 80 to $90^{\circ} \mathrm{C}$ (third generation DHN) and with a building requiring a forward temperature of $65^{\circ} \mathrm{C}$ for its heat distribution.

This scenario was found to already be applicable today and its interest should slightly decrease over time as the thermal grid temperature decreases. Infrastructures of thermal grid networks are costly and complex to develop, especially in city centers where other infrastructures might limit its development. Scenario 2 is thereby proposing an interesting solution to develop the grid capacity while keeping the new infrastructure costs low. In terms of potential, this scenario could enhance the use of low-temperature waste energy or low-temperature renewable energy, and enable replacing fossil fuel-based heating systems with heat from thermal grids. It also has to be noted that Scenario 2 could be coupled with Scenario 1, since it would decrease the return temperature of the grid and enhance the energy that could be valorized by a centralized adHP operating with industrial waste heat as a driving and cold source.

\subsection{Scenario 3: Autonomous adHP with Wood Pellet Burner for Low Energy Demand Buildings}

So far, Scenarios 1 and 2 considered applications for urban areas suitable for DHN. In Scenario 3, the objective is to propose a decentralized solution that could be implemented in rural areas, as shown in Figure 7. This scenario relies on a wood pellet heating system to be used as a driving source and as a backup if necessary. The cold source is coming from the environment (borehole heat exchangers).

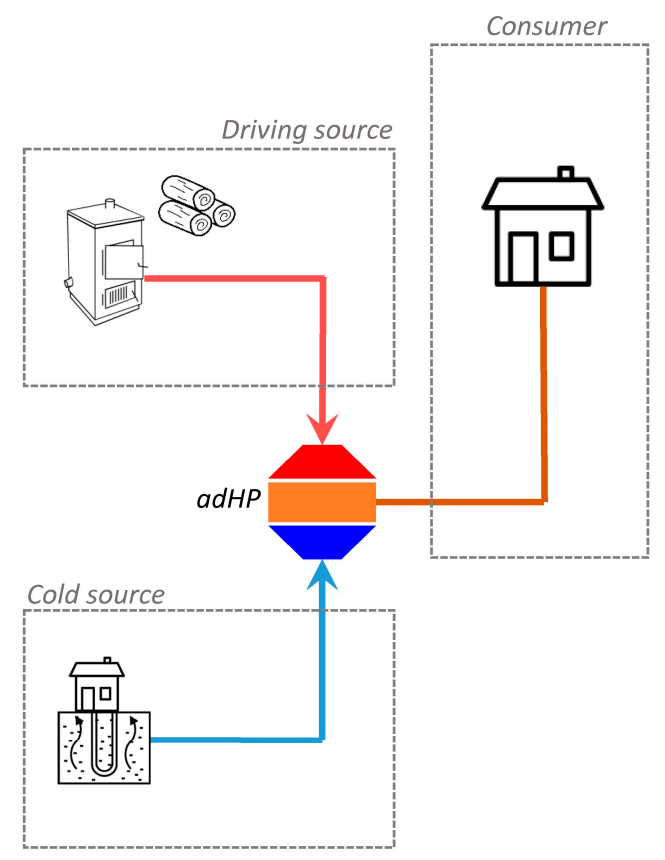

Figure 7. Schematic representation of the Scenario 3.

This scenario is thus an evolution of the actual wood pellet heating system. Scenario 3 was found to be an interesting application for the development of a new generation of high-efficiency wood-based heating systems. It could be especially useful for low-energy buildings with floor heating, and could be an excellent solution for any new construction in rural areas as well as for renovated buildings. Some commercial products have already been developed including this concept but with gas as driving source [6]. The monitored efficiency was found to be promising, approx. 122\% on LHV [27]. 
Scenario 3 is somewhat an adaptation of these commercial systems, but including a renewable energy as driving source.

In this scenario, the temperature triplet for the test phase is $T_{L T}=10{ }^{\circ} \mathrm{C}, T_{M T}=35^{\circ} \mathrm{C}$, and $T_{H T}=$ $81^{\circ} \mathrm{C}$, leading to an estimated COP of 1.44 . This scenario is also of strong interest since it proposes a decentralized system, using the developed adHP without requiring the heavy infrastructure of a district heating network, not economically viable in low energy density territories. Thus, by proposing to couple the wood boiler with the adHP, a new field of interest for the sorption technology is open since rural and urban areas could be considered as potential markets. Recently, two gas fired adsorption heat pumps were available - namely Vitosorp and Zeotherm-from two manufacturers (Viessmann and Vaillant, respectively), the annual efficiency ranges from 1.42 to 1.57 , including a $5 \%$ solar coverage for space heat. Using the developed model of equation (6), we obtained a COP of 1.44, which is in the range of the existing system fired with gas. Therefore, the proposed application appears to be promising and should be investigated further. One identified challenge is the consideration of the specific wood burner dynamics.

\subsection{Scenario 4: Data Centre Cooling Application}

Scenario 4 relies on a cooling application, whereas the three previous scenarios focused on heating applications. This application is more in line with the current use of adHP systems. In this scenario, a data center room is cooled down via an adHP. The heat from the water cooling system for the IT servers is used as driving source and the medium temperature energy is released, to a 4th generation DHN [25] or could be used to regenerate the soil used by a borehole heat exchanger' field, as shown in Figure 8.

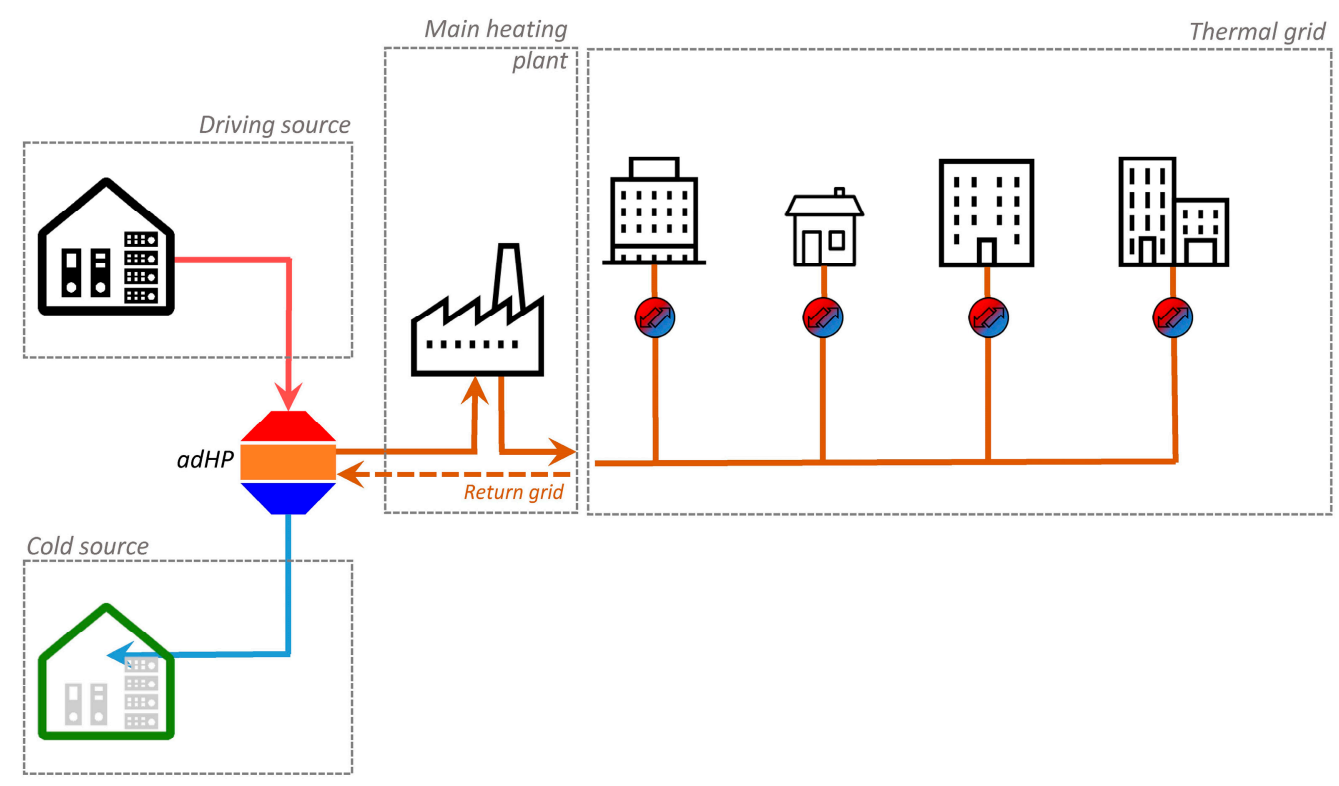

Figure 8. Schematic representation of the scenario 4.

In this scenario, the temperature triplet for the test phase is $\theta_{L T}=18{ }^{\circ} \mathrm{C}, \theta_{M T}=35^{\circ} \mathrm{C}$, and $\theta_{H T}=$ $65^{\circ} \mathrm{C}$, leading to an estimated COP cold of 0.47 . This scenario was found to be interesting but highly dependent on the site and application, requiring a detailed analysis for each individual configuration. The cooling mode refers to the "traditional" applications of the adsorption machines.

\subsection{Summary on Application Scenarios}

In general, the four proposed scenarios present a complementary potential for both present and future applications (short to long term perspectives). These four scenarios are of strong interest in 
order to contribute efficiently to the overall Swiss energy strategy. Several interviews were carried out with industrial partners, particularly regarding thermal grids or incineration plants, and the feedback was found to be positive. Finally, the temperature triplets were set to be representative of one operating point for each scenario. These temperature triplets have been used for the test phase presented hereafter; however, it is clear that, since the space heat demand of the building is evolving because of the external meteorological conditions, times series should also be defined in order to increase the assessment of the adHP performances for each of the four scenarios. Table 4 summarizes the defined temperature triplets as well as the predicted COP for each scenario.

Table 4. Summary of the temperature triplets for the identified scenarios and the estimated COP for heating and cooling applications, respectively.

\begin{tabular}{|c|c|c|c|c|c|}
\hline & $\theta_{L T}$ & $\theta_{M T}$ & $\theta_{H T}$ & $\mathrm{COP}_{h}$ & $\mathrm{COP}_{c}$ \\
\hline Scenario 1 & $16^{\circ} \mathrm{C}$ & $40{ }^{\circ} \mathrm{C}$ & $95^{\circ} \mathrm{C}$ & 1.48 & - \\
\hline Scenario 2 & $48^{\circ} \mathrm{C}$ & $58^{\circ} \mathrm{C}$ & $81.5^{\circ} \mathrm{C}$ & 1.56 & - \\
\hline Scenario 3 & $10^{\circ} \mathrm{C}$ & $35^{\circ} \mathrm{C}$ & $81^{\circ} \mathrm{C}$ & 1.44 & - \\
\hline Scenario 4 & $18^{\circ} \mathrm{C}$ & $35^{\circ} \mathrm{C}$ & $65^{\circ} \mathrm{C}$ & - & 0.47 \\
\hline \multicolumn{4}{|c|}{ THRIVE targets: } & 1.45 & 0.45 \\
\hline
\end{tabular}

\section{Experimental Results}

\subsection{Experimental Plan}

The experimental plan elaborated to validate the performances of the defined scenarios (Table 4) was divided into two phases. During these two test phases, the half cycle time $\tau_{H C}$ was kept constant at $600 \mathrm{~s}$, and the temperature set to point $\theta_{\text {set }}$ of the machine, which was set to its minimum at $6^{\circ} \mathrm{C}$, so that the machine provided the maximum output power. The temperature conditions defined for Scenario 2 are outside the manufacturers recommended range and therefore, while feasible, no insurance was given on the performance of the commercial unit.

Phase 1 aimed at characterizing the performance of the eCoo10 adHP machine for its nominal point, with the three temperatures and flow rates as defined on the manufacturer's plate, except for the MT loop where the maximal flow rate that the test bench could reach is $4500 \mathrm{l} / \mathrm{h}$. Compared to the manufacture recommended flowrate of $5100 \mathrm{l} / \mathrm{h}$, the tested flowrate is $12 \%$ lower. Then the four defined scenarios were tested with the temperature given in Table 4 . These temperatures are considered for simplicity as the inlet temperatures of the corresponding hydraulic circuit.

In phase 2, the performances of the eCoo10 adHP were characterized with reduced flow rates for the same temperatures as before. These reduced flow rates corresponded to $40 \%$ of their nominal value.

The various operating points experimentally tested are summarized in the following Table 5:

Table 5. Summary of the boundary conditions of the experimental plan.

\begin{tabular}{|c|c|c|c|c|c|c|c|c|}
\hline & $\theta_{L T, i n}\left[{ }^{\circ} \mathrm{C}\right]$ & $\theta_{M T, i n}\left[{ }^{\circ} \mathrm{C}\right]$ & $\theta_{H T, i n}\left[{ }^{\circ} \mathrm{C}\right]$ & $\dot{v}_{L T}[1 / \mathrm{h}]$ & $\dot{v}_{M T}[1 / \mathrm{h}]$ & $\dot{v}_{H T}[1 / \mathrm{h}]$ & $\tau_{H C}[\mathbf{s}]$ & $\theta_{\text {set }}\left[{ }^{\circ} \mathrm{C}\right]$ \\
\hline \multicolumn{9}{|c|}{ Phase 1: Nominal Flow Rates } \\
\hline eCoo10 nominal point & 15 & 27 & 85 & & & & & \\
\hline Scenario 1 & 16 & 40 & 95 & & & & & \\
\hline Scenario 2 & 48 & 58 & 81.5 & 2900 & 4500 & 2500 & 600 & 6 \\
\hline Scenario 3 & 10 & 35 & 81 & & & & & \\
\hline Scenario 4 & 18 & 35 & 65 & & & & & \\
\hline \multicolumn{9}{|c|}{ Phase 2: Reduced Flow Rates } \\
\hline eCoo10 nominal point & 15 & 27 & 85 & & & & & \\
\hline Scenario 1 & 16 & 40 & 95 & & & & & \\
\hline Scenario 2 & 48 & 58 & 81.5 & 1160 & 2040 & 1000 & 600 & 6 \\
\hline Scenario 3 & 10 & 35 & 81 & & & & & \\
\hline Scenario 4 & 18 & 35 & 65 & & & & & \\
\hline
\end{tabular}




\subsection{Rated Performances of a Commercial Machine}

Figure 9 presents (a) the rated heating power and (b) the COP for the heating of the eCoo10 tested under the boundary conditions detailed previously. This rated power is calculated as described in Section 2.4.2 over two full cycles, and represents the rate at which the energy at the condenser and adsorber is dissipated. The variation of the rated heating power is large between the scenarios, the nominal condition of the machine-more suited for cooling applications as the return temperature of the MT loop is quite low (e.g., $27^{\circ} \mathrm{C}$ )-has a rated heating power of $20.5 \mathrm{~kW}$. In comparison, the most unfavorable scenario (Scenario 3 ) has a rated heating power of only $8.3 \mathrm{~kW}$. For this scenario, the ratio investment cost for the machine over the heating power is unlikely to be sustainable. Regarding the COP, it varies strongly in function of the external temperature applied to the machine's heat exchangers. Under nominal condition (temperature triplet of 85,27 and $15^{\circ} \mathrm{C}$ ), the COP reaches 1.46 and fulfils the targets. However, the specific scenario shows lower COPs. Thus, improvement of the adsorption bed design and/or use of a different adsorbent is needed.
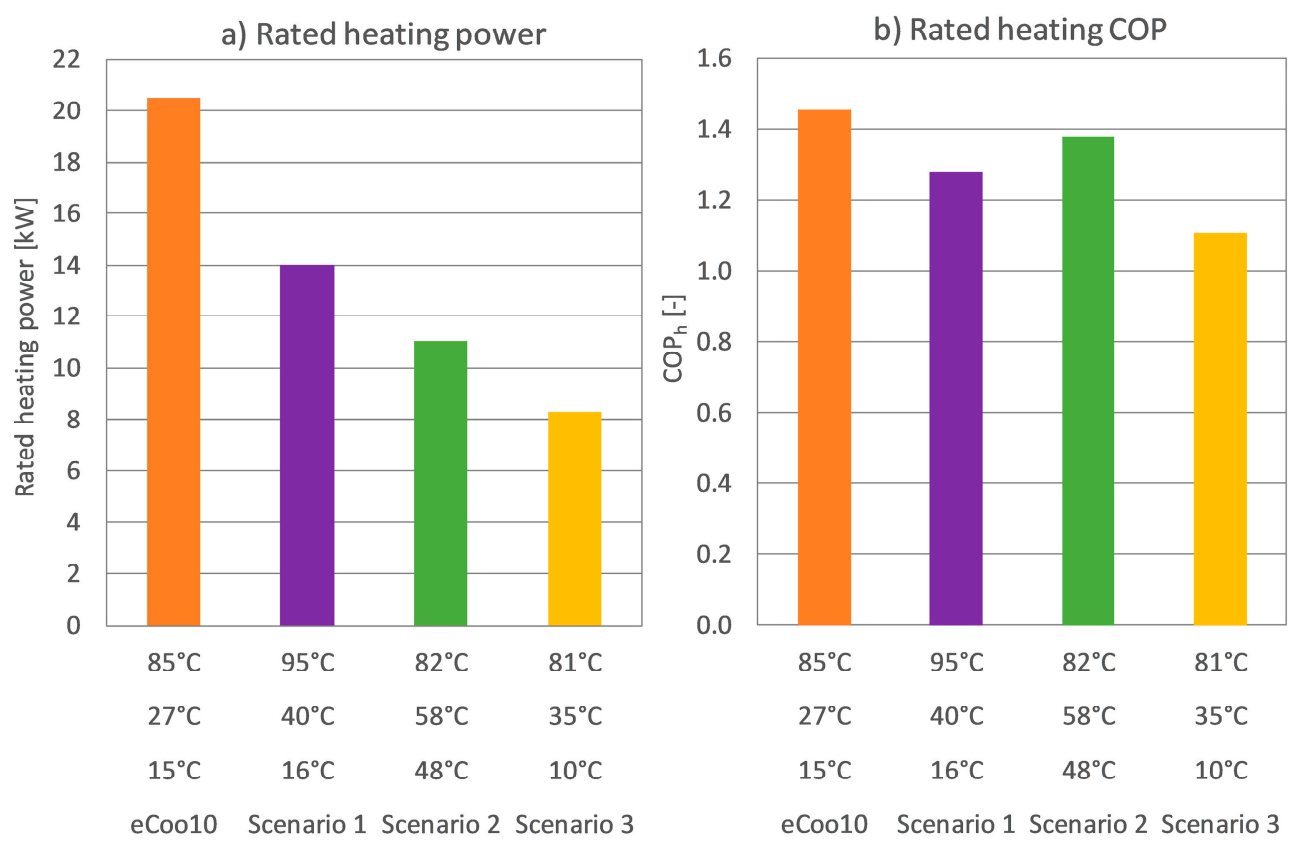

Figure 9. Experimental results at nominal flow rates of the eCoo10 for the heating application scenarios (1-3), (a) Rated heating power and (b) the rated COP for heating.

Reducing the flow rate could be required at part load operation or for applications needing higher temperature differences (i.e., district heating applications). Figure 10 presents the experimental results where the flow rate was reduced to $40 \%$ of the nominal value. It shows that the heating power and COP are degraded up to $38 \%$ and $24 \%$, respectively.

Figure 11 presents (a) the rated cooling power and (b) the cooling COP of the eCoo 10 for the nominal conditions and Scenario 4. The COP at nominal condition falls at 0.48 and is of the same order as the one communicated by the manufacturer. However, the measured performances of Scenario 4 with a COP of 0.24 are very far away from the 0.46 estimated with Equation (6) and presented in Table 4. The rated cooling power of Scenario 4 is also impacted by the temperature lift between the cold source and the recooling source, as well as the lower driving temperature compared to the nominal conditions. The resulting cooling power is decreased by $76 \%$ compared to nominal conditions. The resulting cooling power of $1.7 \mathrm{~kW}$ is very low in comparison with the size, mass and cost of the unit. Further tests would be required in order to identify whether the lower cooling power is induced by the low driving source or the high temperature lift between the MT and LT loop. Some improvement could also come from the optimization of the half cycle time. 

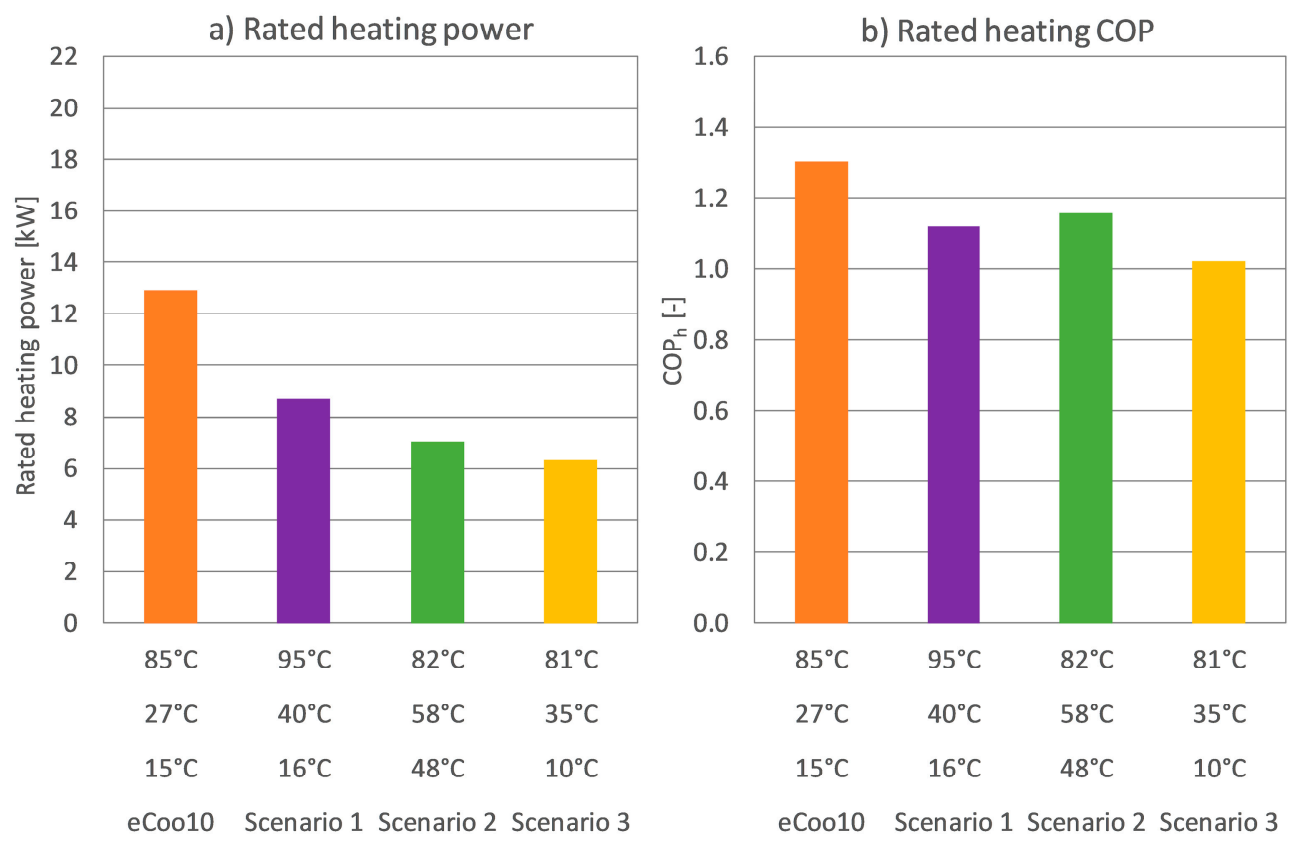

Figure 10. Experimental results at reduced flow rates of the eCoo10 for the heating application scenarios (1-3), (a) Rated heating power and (b) the rated COP for heating.
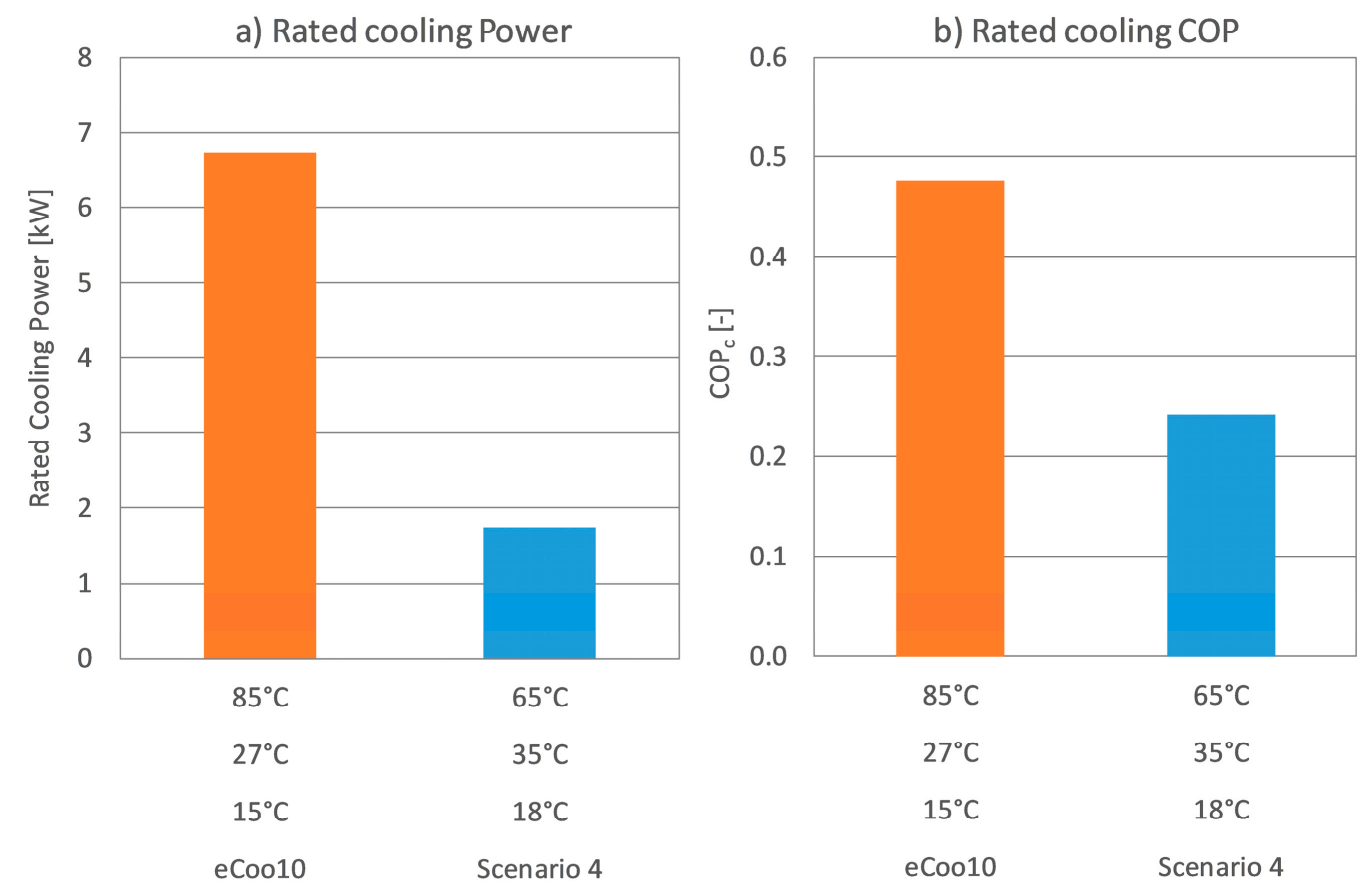

Figure 11. Experimental results of the eCoo10 for the heating application scenarios (1-3), (a) Rated heating power and $(\mathbf{b})$ the rated COP for heating.

\section{Discussion}

\subsection{Evaluation of the Regression Model}

Figure 12 presents the evaluation of the regression models used in this work and given in (6). The model is compared to (1) the literature sample from $[4,20,21]$ which represents the datasets used to fit the parameters of (6); (2) the prediction results of a thermodynamic model developed by Sharonov and Aristov [22], and (3) the experimental results of the eCoo10 presented previously. The regression 
model shows satisfying accuracy with the literature sample, with a correlation coefficient of 0.86 . Compared to the Sharonov and Aristov model, the model takes into account the technical default of a real adsorption machine leading to irreversibilities and, thus, to lower technical efficiencies. They quantify to an average efficiency reduction of around $20 \%$, consequently showing the potential improvement of the design and operation of adHP.

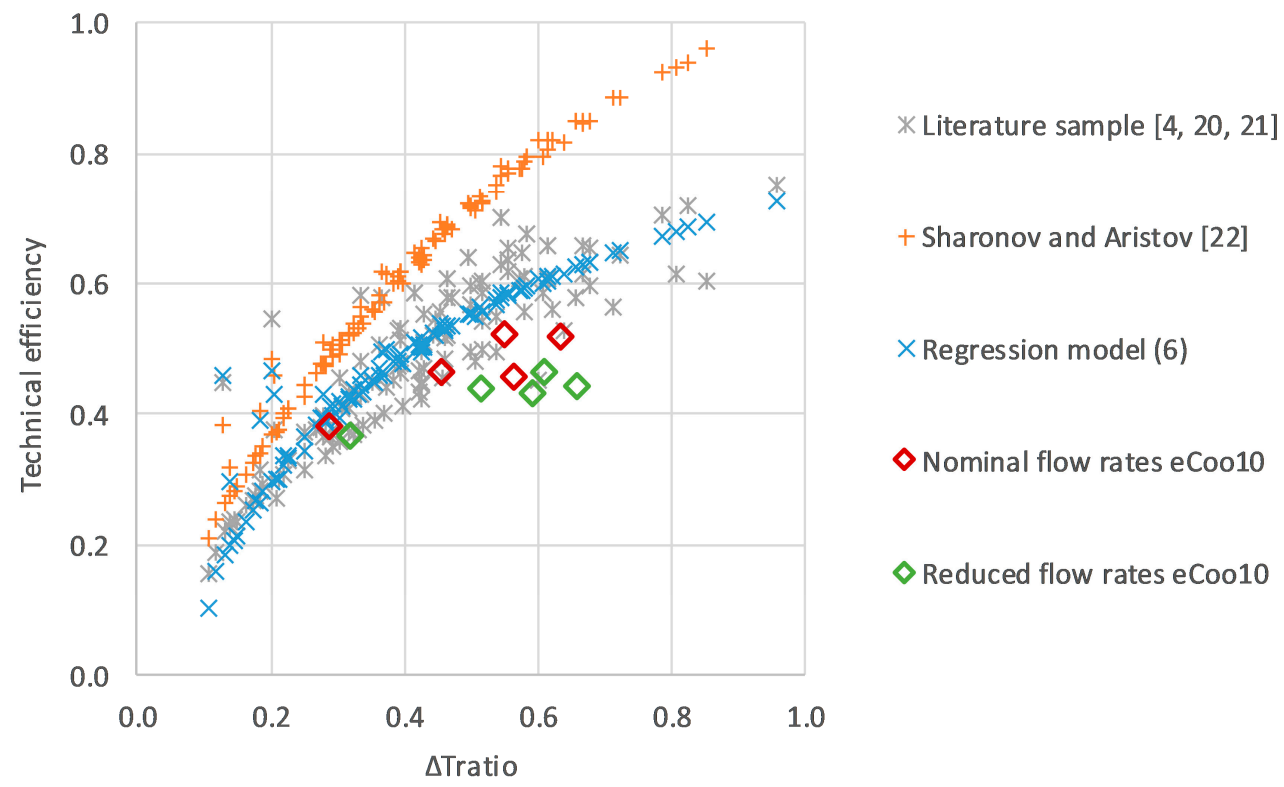

Figure 12. Technical efficiency as a function of the $\Delta T_{\text {ratio }}$ for the literature sample $[4,20,21]$, thermodynamic proposed in [22], the regression model given in (6) and the experimental results of the eCoo10.

The experimental results of the eCoo10 and summarized in Table 6, are lower than the regression model for both nominal and reduced flow rates. This can be explained by the lower flow rate at the MT circuit and the use of glycol/water mixture and has been confirmed by the manufacturer. However, the results at nominal flow rates fall in the range of the literature sample. Moreover, the experimental results at reduced flow rates show that the regression model overestimates the technical efficiency in these very specific conditions. Thus, one should be cautious when estimating the performances of adHP for uncommon applications.

Table 6. Comparison of the experimental results with the model predictions.

\begin{tabular}{|c|c|c|c|c|c|c|c|c|c|}
\hline & \multicolumn{7}{|c|}{ Experimental Results } & \multicolumn{2}{|c|}{ Predictions } \\
\hline & $T_{L T}$ & $T_{M T}$ & $T_{H T}$ & $\Delta T_{\text {ratio }}$ & $C O P_{h}^{c}$ & $C O P_{h}$ & $\eta_{t e c h_{h}}$ & $\eta_{t e c h_{h}}^{*}$ & APE \\
\hline \multicolumn{10}{|c|}{ Phase 1: Nominal Flow Rates } \\
\hline eCoo10 & 287 & 302 & 356 & 0.29 & 3.81 & 1.46 & 0.38 & 0.42 & $9 \%$ \\
\hline Scenario 1 & 291 & 315 & 366 & 0.45 & 2.75 & 1.28 & 0.47 & 0.54 & $15 \%$ \\
\hline Scenario 2 & 321 & 332 & 353 & 0.55 & 2.65 & 1.38 & 0.52 & 0.57 & $10 \%$ \\
\hline Scenario 3 & 284 & 309 & 353 & 0.56 & 2.43 & 1.11 & 0.46 & 0.59 & $29 \%$ \\
\hline Scenario 4 & 292 & 309 & 337 & 0.63 & 2.37 & 1.23 & 0.52 & 0.61 & $18 \%$ \\
\hline \multicolumn{10}{|c|}{ Phase 2: Reduced Flow Rates } \\
\hline eCoo10 & 287 & 303 & 353 & 0.32 & 3.56 & 1.30 & 0.37 & 0.44 & $20 \%$ \\
\hline Scenario 1 & 290 & 315 & 364 & 0.52 & 2.54 & 1.12 & 0.44 & 0.57 & $30 \%$ \\
\hline Scenario 2 & 321 & 333 & 352 & 0.61 & 2.50 & 1.16 & 0.46 & 0.60 & $29 \%$ \\
\hline Scenario 3 & 285 & 310 & 351 & 0.59 & 2.37 & 1.02 & 0.43 & 0.60 & $40 \%$ \\
\hline Scenario 4 & 292 & 309 & 335 & 0.66 & 2.32 & 1.03 & 0.44 & 0.62 & $41 \%$ \\
\hline
\end{tabular}




\subsection{Evaluation and Perspective of the Experimental Results}

All the investigated scenarios show low output power (heating for Scenarios 1, 2 and 3 and cooling for Scenario 4); applying the nominal flow rates would be difficult to justify, because the share of primary energy to drive the hydraulics pump will grow in proportion. Moreover, reducing the nominal flow rate decreases the performance of the machines even more as the heat transfer coefficients deteriorate. This shows the importance of tailoring the adsorber bed and the working to the targeted application in order to upgrade the specific power output of the adHP and-in the end-reduce the bulk, mass and cost of the unit.

Finally, further investigations are needed to identify the key parameters that influence the heating power. The water uptake of the silica gel under the resulting partial pressure and the heat transfer coefficient of the heat exchanger should be thoroughly studied in order to optimize the design of an adsorption heat pump for the specific scenarios considered in this work. Attention should also be given to the optimization of the cycle control to further improve the performances in particular for part load operation.

\section{Conclusions}

In conclusion, a regression model to predict the technical efficiency of adHPs is presented and validated with experimental results of a commercial silica-gel unit. With this model, different integration scenarios ( 3 for heating and 1 for cooling applications) were identified according to thermodynamic and technical constraints setting the boundaries of the exploration domain with a minimum cold source temperature of $0{ }^{\circ} \mathrm{C}$ and a maximum driving temperature of $100^{\circ} \mathrm{C}$. A commercial unit optimized for cooling application was characterized in stationary condition for each scenario. The unit shows performances below the predicted value of the regression model with COP up to $23 \%$ lower for Scenario 3 and up to $48 \%$ for Scenario 4 . However, when the technical efficiencies of the adHP are compared with the data found in the literature, the experimental results are in the lower range-indicating that some improvements are possible. Consequently, further investigation in terms of heat and mass exchanger design as well as new working pairs are needed to close the gap between actual adHP and the thermodynamics limitation of a trithermal machine.

Author Contributions: Conceptualization, P.P., D.P. and X.J.; methodology, P.P. and X.J.; validation, X.J.; formal analysis, P.P. and X.J.; investigation, P.P and X.J.; resources, X.J., P.P. and M.G.; data curation, X.J., P.P. and M.G..; writing—original draft preparation, X.J., P.P, M.G. and A.D.; writing—review and editing, X.J., A.D. and D.P.; visualization, X.J., P.P. and M.G.; supervision, D.P.; project administration, D.P.; funding acquisition, A.D. All authors have read and agreed to the published version of the manuscript.

Funding: This research was funded by Swiss National Science Foundation, National Research Program 70 grant number 407040153949.

Acknowledgments: The authors would like also to thank Patrick Ruch, for his relevant inputs throughout the THRIVE project, Cyril Mahmed for his help on the test bench and the experimental work and Guy Wagner for the drawing of figure 5. All building icons by Creative Stall from the noun project, metal oven by Sergey Novosyolov from the noun project, wood logs by Vector Points from the noun project and all other icons and figures by the authors.

Conflicts of Interest: The authors declare no conflict of interest. The funders had no role in the design of the study; in the collection, analyses, or interpretation of data; in the writing of the manuscript, or in the decision to publish the results.

\section{List of Acronyms}

The following acronyms are used in this manuscript:

$\begin{array}{llll}\text { AdHP } & \text { Adsorption heat pump } & \text { HTF } & \text { Heat transfer fluid } \\ \text { COP } & \text { Coefficient of performance } & \text { MT } & \text { Medium temperature } \\ \text { DHN } & \text { District heating network } & \text { LT } & \text { Low temperature } \\ \text { EER } & \text { Energy efficiency ratio } & \text { LHV } & \text { Low heating value } \\ \text { HT } & \text { High temperature } & \text { THRIVE } & \begin{array}{l}\text { Thermally driven adsorption heat pumps for } \\ \text { substitution of electricity and fossil fuels }\end{array}\end{array}$




\section{List of Notations}

The following acronyms are used in this manuscript:

\begin{tabular}{llll}
\multicolumn{2}{l}{ Notations } & \multicolumn{2}{l}{ Subscripts and superscripts } \\
$c p$ & specific heat, $\mathrm{kJ} /(\mathrm{kg} \mathrm{K})$ & $L T$ & low temperature \\
$\dot{v}$ & volume flow rate, $\mathrm{l} / \mathrm{h}$ & $\mathrm{MT}$ & medium temperature \\
$T$ & temperature, $\mathrm{K}$ & $H T$ & high temperature \\
$t$ & time, s & $2 \mathrm{C}$ & two consecutive cycle \\
$\dot{Q}$ & thermal power, $\mathrm{kW}$ & $h$ & heating \\
$Q$ & thermal energy, $\mathrm{kWh}$ & $\mathrm{C}$ & cooling \\
Greek symbols & $\mathrm{C}$ & Carnot \\
$\eta \quad$ efficiency & $H C$ & half cycle \\
$\theta \quad$ Temperature,${ }^{\circ} \mathrm{C}$ & tech & technical \\
$\rho \quad$ density, $\mathrm{kg} / \mathrm{m}^{3}$ & $*$ & Predicted \\
$\tau$ & cycle time, $\mathrm{s}$ & &
\end{tabular}

\section{References}

1. Prognos, A.G.; Infras, A.G.; TEP Energy GmbH. Analyse des Schweizerischen Energieverbrauchs 2000-2012 Nach Verwendungszwecken; SFOE: Bern, Switzerland, 2013.

2. Statistique Globale Suisse de L'énergie; SFOE: Bern, Switzerland, 2011.

3. Prognos, A.G. Die Energieperspektiven für die Schweiz bis 2050-Energienachfrage und Elektrizitätsangebot in der Schweiz 2000-2050-Ergebnisse der Modellrechnungen für das Energiesystem; SFOE: Bern, Switzerland, 2012.

4. Demir, H.; Mobedi, M.; Ülkü, S. A review on adsorption heat pump: Problems and solutions. Renew. Sustain. Energy Rev. 2008, 12, 2381-2403. [CrossRef]

5. Faraday, M. On the condensation of several gases into liquids. Philos. Trans. R. Soc. Lond. 1823, 113, $189-198$.

6. Dawoud, B. Viessmann gas driven sorbtion heat pumps. In Proceedings of the Gas Heat Pumps Workshop, Saint-Denis/Paris, France, 1-2 December 2011.

7. Tiemeier, H. Vaillant systems with zeoTHERM VAS 106/4. In Proceedings of the Gas Heat Pumps Workshop, Saint-Denis/Paris, France, 1-2 December 2011.

8. Fan, Y.; Luo, L.; Souyri, B. Review of solar sorption refrigeration technologies: Development and applications. Renew. Sustain. Energy Rev. 2007, 11, 1758-1775. [CrossRef]

9. Aristov, Y.I. Adsorptive transformation of ambient heat: A new cycle. Appl. Therm. Eng. 2017, 124, 521-524. [CrossRef]

10. Tokarev, M. A Double-Bed Adsorptive Heat Transformer for Upgrading Ambient Heat: Design and First Tests. Energies 2019, 12, 4037. [CrossRef]

11. Freni, A.; Maggio, G.; Sapienza, A.; Frazzica, A.; Restuccia, G.; Vasta, S. Comparative analysis of promising adsorbent/adsorbate pairs for adsorptive heat pumping, air conditioning and refrigeration. Appl. Therm. Eng. 2016, 104, 85-95. [CrossRef]

12. Ammann, J.; Michel, B.; Studart, A.R.; Ruch, P.W. Sorption rate enhancement in SAPO-34 zeolite by directed mass transfer channels. Int. J. Heat Mass Transf. 2019, 130, 25-32. [CrossRef]

13. Li, Y.X.; Wang, L.; Yuan, Z.X.; Chen, Q.F. Enhancement of heat transfer in adsorption bed of vacuum-tube with fins. Appl. Therm. Eng. 2019, 153, 291-298. [CrossRef]

14. Wittstadt, U.; Füldner, G.; Laurenz, E.; Warlo, A.; Große, A.; Herrmann, R.; Schnabel, L.; Mittelbach, W. A novel adsorption module with fiber heat exchangers: Performance analysis based on driving temperature differences. Renew. Energy 2017, 110, 154-161. [CrossRef]

15. Quiquerez, L.; Cabrera Santelices, J.D.; Hollmuller, P.; Lachal, B.M. Températures de Distribution de Chauffage du Parc Immobilier Genevois; Partenariat SIG - UNIGE: Genève, Switzerland, 2013.

16. Aristov, Y.I. Chemical and adsorption heat pumps: Cycle efficiency and boundary temperatures. Theor. Found. Chem. Eng. 2008, 42, 873-881. [CrossRef]

17. Alefeld, G.; Radermacher, R. Heat Conversion Systems; CRC Press: Boca Raton, FL, USA, 1994; ISBN 9780849389283.

18. Tozer, R.M.; James, R.W. Fundamental thermodynamics of ideal absorption cycles. Int. J. Refrig. 1997, 20, 120-135. [CrossRef] 
19. Pesaran, A.; Lee, H.; Hwang, Y.; Radermacher, R.; Chun, H.-H. Review article: Numerical simulation of adsorption heat pumps. Energy 2016, 100, 310-320. [CrossRef]

20. Meunier, F. Adsorption heat powered heat pumps. Appl. Therm. Eng. 2013, 61, 830-836. [CrossRef]

21. Wang, D.C.; Shi, Z.X.; Yang, Q.R.; Tian, X.L.; Zhang, J.C.; Wu, J.Y. Experimental research on novel adsorption chiller driven by low grade heat source. Energy Convers. Manag. 2007, 48, 2375-2381. [CrossRef]

22. Sharonov, V.E.; Aristov, Y.I. Chemical and adsorption heat pumps: Comments on the second law efficiency. Chem. Eng. J. 2008, 136, 419-424. [CrossRef]

23. Melograno, P.N.; Vasta, S.; Boudehenn, F.; Fedrizzi, R.; Doell, J. Final Deliverable Subtask A1-Chiller Characterization. IEA SHC Task 482015.

24. BIPM; IEC; IFCC; ILAC; ISO; IUPAC; IUPAP. Evaluation of Measurement Data-Guide to the Expression of Uncertainty in Measurement; JCGM: Sèvre, France, 2008; ISBN 9267101889.

25. Lund, H.; Werner, S.; Wiltshire, R.; Svendsen, S.; Thorsen, J.E.; Hvelplund, F.; Mathiesen, B.V. 4th Generation District Heating (4GDH): Integrating smart thermal grids into future sustainable energy systems. Energy 2014, 68, 1-11. [CrossRef]

26. Zhang, Y.; Shi, W.; Zhang, Y. From heat exchanger to heat adaptor: Concept, analysis and application. Appl. Energy 2014, 115, 272-279. [CrossRef]

27. Critoph, R.E. State of the Art in Gas Driven Heat Pumps; University of Warwick: Coventry, UK, 2013.

(C) 2020 by the authors. Licensee MDPI, Basel, Switzerland. This article is an open access article distributed under the terms and conditions of the Creative Commons Attribution (CC BY) license (http://creativecommons.org/licenses/by/4.0/). 